\title{
SPECTRAL OPERATORS IN SPACES OF DISTRIBUTIONS
}

\author{
BY H. H. SCHAEFER AND B. J. WALSH ${ }^{1}$ \\ Communicated by Einar Hille, April 18, 1962
}

The purposes of this note are to complement the results of [4] by citing examples of spectral operators (in the sense of that paper) on non-normable locally convex spaces and to announce a result on the structure of spectral operators which indicates that these examples exhibit rather typical behavior for spectral operators on a large class of locally convex spaces of interest to analysts.

We retain the notions of [4] and refer to it for proofs. Let $E$ be a locally convex space (always assumed Hausdorff), $\mathfrak{L}(E)$ the algebra of continuous linear transformations of $E$ into itself (the identity being denoted by $e), \mathfrak{L}_{s}(E)$ the algebra $\mathfrak{L}(E)$ under the topology of pointwise convergence, and let $\mathfrak{L}_{\sigma}(E)$ be $\mathcal{L}(E)$ under the weak topology associated with $\mathfrak{L}_{s}(E)$. If $u$ is a linear transformation defined on a dense vector subspace $D_{u}$ of $E$ with values in $E$, the resolvent set of $u$ (denoted by $\rho(u)$ ) is the set of all complex numbers $\lambda$ possessing neighborhoods $N_{\lambda}$ such that for each $\mu \in N_{\lambda}$, the operator $\mu e-u$ is 1-1 and has range dense in $E$, $(\mu e-u)^{-1}$ has a (necessarily unique) extension $R(\mu) \in \mathfrak{L}(E)$ and $R(\cdot)$ is holomorphic in $N_{\lambda}$-to these we adjoin the point $\infty$ if $R(\cdot)$ is holomorphic in a neighborhood of $\infty$. The spectrum of $u$ (denoted by $\sigma(u)$ ) is the complement of $\rho(u)$ in the Riemann sphere; the point spectrum $\pi(u)$ is the set of complex numbers $\lambda$ for which $\lambda e-u$ is not $1-1$.

Given a compact Hausdorff space $X$, let $\mathrm{e}(X)$ denote its algebra of continuous complex-valued functions and $B(X)$ its algebra of complex-valued bounded Baire functions, both under the supremum norm. A spectral measure is a mapping $\delta \rightarrow \mu(\delta)$ from the $\sigma$-algebra of Baire subsets of $X$ to $\mathscr{L}(E)$, countably additive in $\mathscr{L}_{\sigma}(E)$ and satisfying $\mu(X)=e, \mu\left(\delta_{1} \cap \delta_{2}\right)=\mu\left(\delta_{1}\right) \cdot \mu\left(\delta_{2}\right)$ for any two Baire sets $\delta_{1}$ and $\delta_{2}$. Under suitable hypotheses (e.g., $E$ weakly semi-complete [= weakly sequentially complete] and tonnelé) every spectral measure $\mu$ defines a continuous homomorphism $\phi$ of $B(X)$ into $\mathscr{L}_{s}(E)$; conversely, any continuous homomorphism $\phi$ of $\mathfrak{e}(X)$ into $\mathfrak{L}_{\sigma}(E)$ has associated with it a spectral measure $\mu$ (and thus an extension to $B(X)$ ). The connection between the measure and the homomorphism is given by

$$
\phi(f)=\int f d \mu .
$$

\footnotetext{
1 National Science Foundation Cooperative Graduate Fellow.
} 
An operator of the form $\int f d \mu$ for some spectral measure $\mu$, compact Hausdorff space $X$ and bounded Baire function $f$ on $X$ will be called a spectral operator (with bounded spectrum) in $\mathfrak{L}(E)$. If $u=\int f d \mu$ is a spectral operator with bounded spectrum, then $\sigma(u) \subset[f(X)]^{-}$and there exists a unique spectral measure $\nu$ on $\sigma(u)$ for which $u=\int \lambda d \nu(\lambda)$ : specifically, $\nu=\mu \circ f^{-1}$.

Spectral operators with unbounded spectrum are defined similarly; these are operators $v: x \rightarrow \int f d \mu(x)$ where $f$ is a Baire function not $\mu$ essentially bounded, and have as domain the vector subspace $D_{v}$ of $E$ for whose elements $x$ the function $f$ is $\mu(\cdot) x$-integrable. $D_{v}$ is always dense in $E$ and $v$ is always closed. In this case also $\sigma(v)$ $C[f(X)]^{-}$, and there exists a spectral measure $\nu$ on $\sigma(v)$ for which $v x=\int \lambda d \nu(\lambda) x$ for all $x \in D_{v}$. (Here $\nu$ is known to be unique if $\rho(v)$ is nonempty or if $E$ is a Hilbert space: whether $\nu$ is unique in other cases remains an open problem.) When $E$ is a Banach space, spectral operators with unbounded spectrum are never defined on all of $E$ and never elements of $\mathcal{L}(E)$; however, they can have both these properties in the non-normable case (see Theorem 1 below). For Banach spaces, these notions of spectral operators agree with Dunford's concept of spectral operators of scalar type. (For an account of the intensive research on spectral operators on Banach spaces carried on by Dunford, Badé and J. Schwartz inter aliis, see [1].)

THEOREM 1. The following operators are spectral on the spaces indicated:

(a) $x \rightarrow f * x$ on the spaces $D_{T^{n}}^{n}$ and $D_{T^{n}}^{\prime n}$ of $C^{\infty}$ functions and distributions respectively on the $n$-dimensional torus $T^{n}[5$, Tome 2, p. 80]. Here * denotes convolution and $f$ may be any element of $\mathfrak{D}_{T^{n}}^{\prime}$; in particular, differential operators with constant coefficients are spectral on $D_{T^{n}}{ }^{n}$ and $\mathfrak{D}_{T}^{\prime}$.

(b) $x \rightarrow d(p \cdot d x / d t) / d t+q \cdot x$ on $\mathscr{D}_{T^{1}}$ and $\mathscr{D}_{T^{1}}^{\prime}$ : here $p$ and $q$ are realvalued $C^{\infty}$ functions on the 1-dimensional torus $T^{1}$.

(c) $x \rightarrow-d^{2} x / d t^{2}+t^{2} \cdot x$ on the spaces $\mathrm{S}$ and $\mathrm{S}^{\prime}$ of rapidly decreasing $C^{\infty}$ functions and temperate distributions (respectively) on the real line; thus the Fourier transform $x(t) \rightarrow \int e^{-2 \pi i u t} x(u) d u$ (considered as an automorphism of $\$$ ) and its adjoint (considered as an automorphism of $\mathrm{s}^{\prime}$ ) are spectral.

(d) Any operator on $\omega_{d}$ or $\phi_{d}$ which possesses a diagonal matrix with respect to the coördinates ( $\omega_{d}$ and $\phi_{d}$ being the product and locally convex direct sum respectively of $d$ copies of the scalar field).

In each of the cases (a), (b) and (c), the spectrality of the operator is a corollary of the fact that the eigenfunctions of a classical boundary-value problem associated with the operator form absolute bases 
in $D_{T^{n}}$ or $S$ respectively: in these spaces, an operator possessing a diagonal matrix with respect to some absolute basis is always spectral (see $\left[4\right.$, pp. 161, 170]). The reasoning in case (d) is similar. ${ }^{2}$

THEOREM 2. Let $E$ be a reflexive nuclear space and $u$ be a spectral operator on $E$. Then $\pi(u)$ is nonempty, and in fact dense in $\sigma(u)$. Moreover,

$$
u x=\sum_{\lambda \in \pi(u)} \lambda \nu(\{\lambda\}) x, \quad x \in D_{u},
$$

where $\nu$ is the spectral measure on $\sigma(u)$, unique except possibly when $\sigma(u)$ is the entire Riemann sphere, for which

$$
u x=\int \lambda d \nu(\lambda) x, \quad x \in D_{u} .
$$

This class of spaces includes the spaces $D, D^{\prime}, \mathcal{E}, \mathcal{E}^{\prime}, D_{T^{n}}, D_{T^{n}}^{\prime}, \mathcal{S}$ and $S^{\prime}$ of L. Schwartz ([5]; see [2, Chapter 2, p. 54 ff.] for proof that these spaces are nuclear), as well as the spaces $H(G)$ of holomorphic functions on domains $G$ of the complex plane (see e.g. [3, p. $375 \mathrm{ff}$.]). Thus the spectral operators on these spaces are of a more severely restricted variety than might at first be supposed. For example, Theorem 2 implies that the operators $x \rightarrow f * x$ and $x \rightarrow g \cdot x$ on $S$, where $f \in \mathcal{O}_{C}^{\prime}$ and $g \in \mathcal{O}_{M}$, are spectral only in the trivial cases where $f=\lambda \delta_{0}$ or $g=\lambda$, i.e., when the operator is a scalar multiple of the identity. These stringent conditions on the spectrum also rule out spectrality for a large number of differential operators on the spaces $S$ and $\boldsymbol{S}^{\prime}$. Proofs of these results will appear elsewhere.

\section{REFERENCES}

1. N. Dunford, $A$ survey of the theory of spectral operators, Bull. Amer. Math. Soc. 64 (1958), 217-274.

2. A. Grothendieck, Produits tensoriels topologiques et espaces nucléaires, Mem. Amer. Math. Soc. No. 16 (1955), 191+140 pp. 1960.

3. G. Köthe, Topologische lineare Räume. I, Springer, Berlin-Göttingen-Heidelberg,

4. H. Schaefer, Spectral measures in locally convex algebras, Acta Math. 107 (1962), $125-173$.

5. L. Schwartz, Théorie des distributions, Tomes 1 et 2, Hermann, Paris, 19501951.

UNIVERSITY OF MiCHIGAN AND UNIVERSITÄT TÜBINGEN

${ }^{2}$ It can be shown that all spectral operators on the spaces $\omega_{d}$ and $\phi_{d}$ are similar to those of case (d): given a spectral operator $u$ on $\omega_{d}$, there exists an automorphism $v$ of $\omega_{d}$ for which $v u v^{-1}$ has a diagonal matrix with respect to the coordinates of $\omega_{d}$, and similarly for $\phi_{d}$. Thus the structure of spectral operators on these spaces for $d \geq \boldsymbol{N}_{0}$ agrees with that obtained in the familiar case when $d$ is finite. 